\title{
Novel approach of Heat Rate Enhancement in a Rectangular channels with thin Porous Layer at the channel walls
}

\author{
M.Z. Saghir*
}

Ryerson University, dept of mechanical and Industrial Engineering, Toronto, Canada

*Corresponding email: zsaghir@ryerson.ca

\begin{abstract}
Heat enhancement is a topic of great interest nowadays due to its different application in industries. Porous material also known as metallic foam plays a major role in heat enhancement at the expenses of pressure drop. Flow in channels demonstrate the usefulness of this technology in heat extraction. In our current study, a porous strip attached to the channels walls is proposed as an alternative for heat enhancement. The thickness of the porous strip was varied for different Reynolds number. By maintaining laminar regime and using water as fluid, we determined an optimum thickness of porous material leading to the highest performance evaluation criterion. In our current study with the aspect ratio being the porous strip thickness over the channel width, an aspect ratio of 0.2 is found to be the alternative. A $40 \%$ increase in heat enhancement is detected in the presence of porous strip when compared to a clear channel case for a Reynolds number equal to 200 and improve further as the Reynolds number increase accordingly.
\end{abstract}

Keyword: Rectangular channels, Twisted tape inserts, Laminar flow, Forced convection, Water, Heat enhancement, Pressure drop, Performance evaluation criteria

\section{Introduction}

Heat enhancement and heat storage are amongst the most studied topics in heat transfer. Ways to improve heat extraction received a large number of interests amongst researchers in this field [1]. Bayomy [2] in his Ph D study investigated experimentally and numerically porous rectangular channels and determined the thermal efficiency for different channel configuration. In one hand porous media help heat extraction but at the expenses of pressure drop. Welsford et al [3] and Delisle et al [4] investigated numerically and experimentally the use of nanofluid with different nanoparticles concentration as a mean to enhance the heat extraction. They demonstrated that such approach is feasible but the pressure drop is noticeable as well. Plant et al [5] extended the previous work by using hybrid fluid. In their experimental and numerical study, they demonstrated that adding copper help enhance further the heat extraction but the pressure drops keep increasing in magnitude thus more pumping power is needed. Different metallic foam permeabilities were used aiming at improving the thermal efficiency. Additional studies took place by other researchers within Saghir group [6-10]

One of the major problems in using nanoparticles is the sedimentation in the bottom of the channels making the pores in porous material blocked thus defeating the purpose of using nanoparticles. Saghir and Rahman [11] were able to investigate this problem by 
including the Brownian motion and the thermophoretic effect. They were able to demonstrate an optimum flow rate to avoid having sedimentation in the channel. However, an innovative new approach which in one hand improve the heat extraction and do not affect the pressure drop is proposed in the present paper.

Investigation of thin porous layer around a pipe has been investigated by Ghadikolaie et al [12]. An increase in heat transferred rate of $20 \%$ has been reported. Similar work by T'Joen et al [13] is conducted where they simulated a row of aluminum tubes which were wrapped with a thin layer of metallic foam. Their findings confirm that the existence of this foam layer help increase the heat transfer rate. In line with the numerical work stated earlier, Al Salem et al [14] conducted an experimental study of forced convection over a heated tube wrapped with a porous layer at the exterior wall. The presence of porous layer augmented the heat transfer without affecting the pressure drop. Le Breton et al [15] used a thin porous layer at the wall of a square cavity in the presence of natural convection. Their finding suggest that the porous layer reduced the upwind flow and lead to a decrease in the convective heat transfer. A lack of investigation of thin porous layer inside a square channel lead the author to investigate this problem numerically and then experimentally.

In this paper we propose an innovative and novel numerical approach in adding to a free channel walls a thin layer of porous foam. The goal is to continue to improve the heat enhancement without affecting the pressure drop. The novelty of the work is to be able to determine an optimum porous layer thickness to achieve the stated goal. Section 2 discuss the problem description followed by section 3 where the finite element formulation and the applied boundary conditions are discussed. To demonstrate the accuracy of the model, a comparison with experimental data is presented in section 4. Section 5 presents the results obtained followed by the conclusion in section 6 .

\subsection{Problem descriptions}

In the present study, efforts are made to investigate the importance of using different porous inserts in a rectangular channel. Enhancing heat removal by not disturbing the pressure drop is the main aim of this paper. Four different cases have been investigated in this paper. Figure 1 presents the model under investigation. This model has been investigated numerically and experimentally by different researchers [1-5] in the absence of porous foam or fully filled with porous foam. In the present study, the channels are plain channels and a porous metallic foam strip having different thickness have been attached at its walls. In the previous study by Plant et al [6], they demonstrated numerically and experimentally that nanofluid achieved a heat enhancement better than water. 


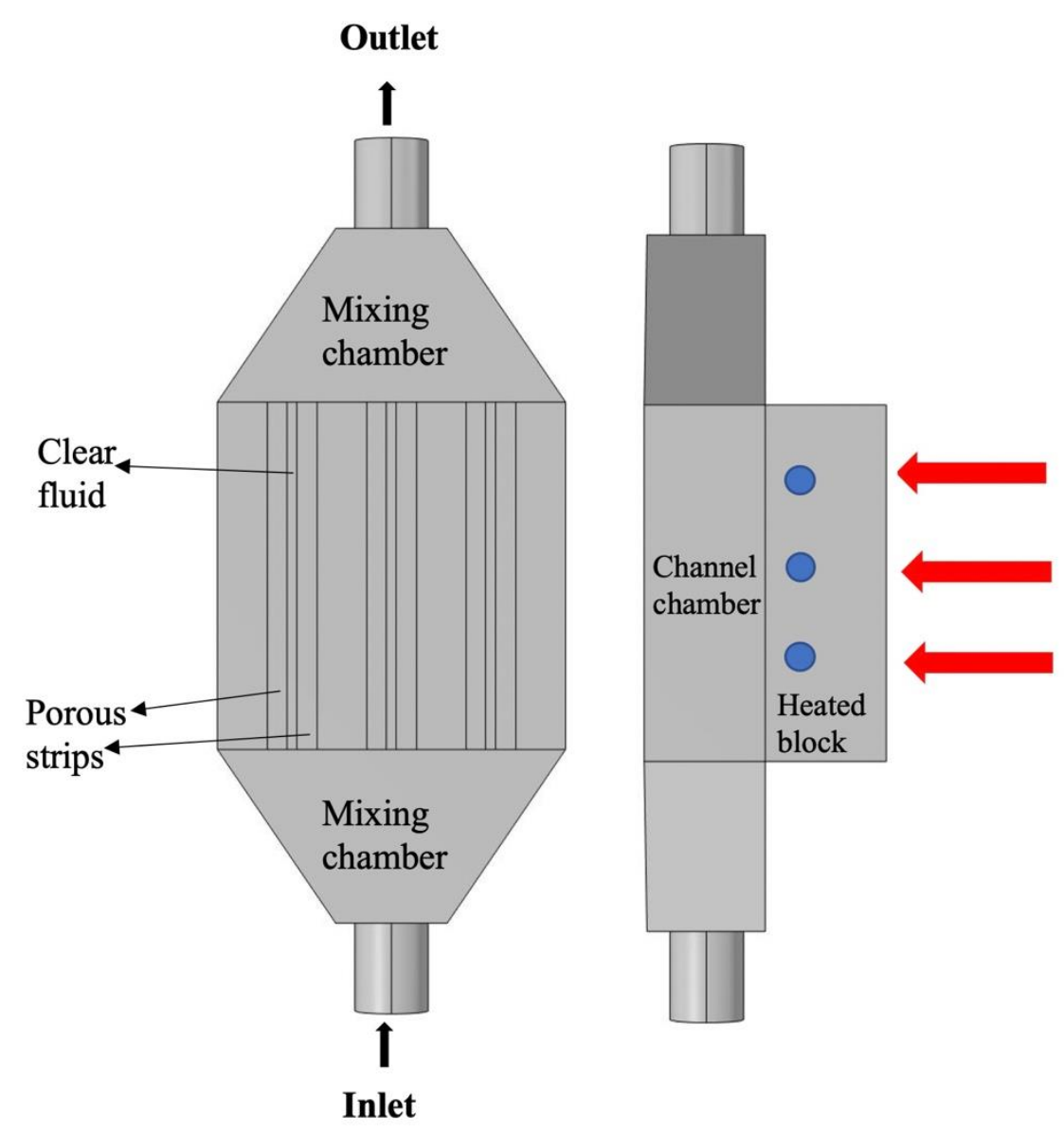

Figure 1. Problem description

However, nanofluid create a larger pressure drops than water. It is the focus in this paper to figure out a means to improve heat extraction and maintaining a lower pressure drop by using water as the circulating fluid.

In Figure 1, two mixing chambers are present at the inlet and at the outlet and the flow enters at a certain temperature Tin and certain flow rate. Temperature is measured 1 $\mathrm{mm}$ below the interface (see the blue dot) and the thermocouple are located at the middle of the heated block made of Aluminum. The channel size is $0.00535 \mathrm{~m}$ in width and 0.0127 $\mathrm{m}$ in height. The block size containing the three channels, identified as channel chambers, is a square block of $0.0375 \mathrm{~m}$ in side made of Aluminum as well. Red arrows show the heated element location where a heat flux is applied to the adjacent metallic block.

At each channel, a strip of porous layer is added as shown in Figure 1. The strip is attached at the three walls surfaces of the corresponding channel, mainly the bottom side and the two left and right side of the channel. The porous foam is made of identical material as the channel which is Aluminum. Figure 2 presents the studied configuration for some aspect ratio. The aspect ratio is the ratio of the strip thickness over the width of the channel which is maintained constant and equal to $0.00535 \mathrm{~m}$. As the aspect ratio increase the porous strip thickness increase accordingly. Thus, for an aspect ratio of 0.5 the channel 
is completely porous and for an aspect ratio equal to zero means it is a clear fluid in a channel with no porous strip. With such configurations, in the presence of porous strip, the flow will circulate in two places inside the channel. In the first place, flow penetration will be in the porous strip where the boundary and thermal layers are formed and the second flow is in the clear path where no porous strip. Thus, as the aspect ratio increase from 0.1 to 0.5 , the flow in free channel is reduced and more flow penetration exist in the porous area.

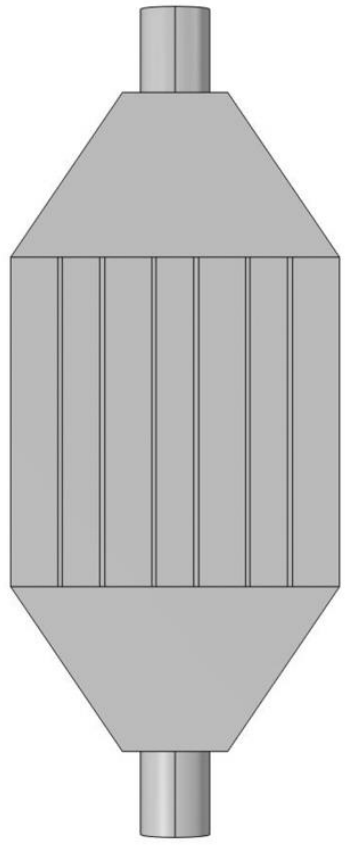

a) $\mathrm{AR}=\mathbf{0 . 1}$

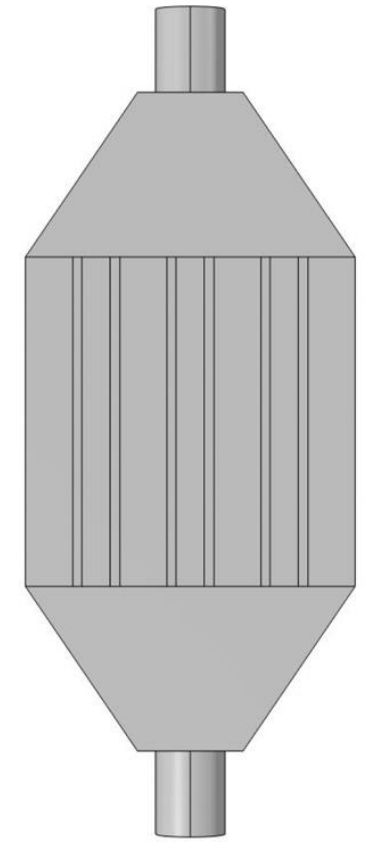

b) $\mathrm{AR}=0.2$

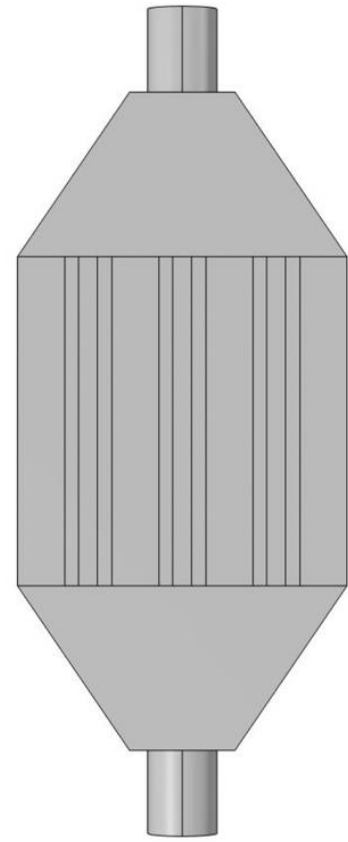

c) $\mathrm{AR}=\mathbf{0 . 3}$

Figure 2. Models with different aspect ratio

The reason for introducing this proposed design of porous strip is to be able to investigate the heat enhancement in a channel by reducing the large pressure drop observed experimentally and numerically. The flow is assumed to be laminar with Reynolds number not exceeding 600. The questions which we raised from this analysis are firstly would the existence of porous strip lead to a better performance evaluation criterion than in the case of a porous channels or a clear channels and secondly whether it exist an optimum aspect ratio or porous thickness for high heat enhancement. These two questions will be addressed in the discussion section

\subsection{Finite element formulations and boundary condition}

The full Navier-Stokes equation together with the energy equation were solved numerically using the finite element code COMSOL [16]. In addition, the heat conduction equation was also solved for the solid part of the model which is mainly the channel walls as well as the heated block. Below we present the formulation in non-dimensional form;

\subsection{Fluid flow formulation}


Using the following non-dimensional parameter

$X=\frac{x}{D}, Y=\frac{y}{D}, Z=\frac{z}{D}, U=\frac{u}{u_{\text {in }}}, V=\frac{v}{u_{\text {in }}}, W=\frac{w}{u_{\text {in }}}, P=\frac{p D}{\mu u_{\text {in }}}, \theta=\frac{\left(T-T_{\text {in }}\right) k}{q " D}$

The non-dimensional terms obtained are;

$\operatorname{Re}=\frac{\rho \mathrm{u}_{\mathrm{in}} \mathrm{D}}{\mu}$, and $\operatorname{Pr}=\frac{\mathrm{cp} \mu}{\mathrm{k}}$

Here $u_{\text {in }}$ is the velocity at the inlet as shown in Figure 1. The Characteristic Length D is taken equal to $0.01897 \mathrm{~m}$, and $\mathrm{Re}$ and $\mathrm{Pr}$ are the Reynolds number and the Prandtl number of the fluid which is water in the present study. The physical properties of the water are taken from the literature at room temperature.

The full Navier-Stokes equation in three dimensional are as follows

$X$ direction

$\operatorname{Re}\left[U \frac{\partial U}{\partial X}+V \frac{\partial U}{\partial \mathrm{Y}}+W \frac{\partial U}{\partial \mathrm{Z}}\right]=-\frac{\partial \mathrm{P}}{\partial \mathrm{X}}+\left[\frac{\partial^{2} \mathrm{U}}{\partial \mathrm{X}^{2}}+\frac{\partial^{2} \mathrm{U}}{\partial \mathrm{Y}^{2}}+\frac{\partial^{2} \mathrm{U}}{\partial \mathrm{Z}^{2}}\right]$

$Y$ direction

$\operatorname{Re}\left[\mathrm{U} \frac{\partial \mathrm{V}}{\partial \mathrm{X}}+\mathrm{V} \frac{\partial \mathrm{V}}{\partial \mathrm{Y}}+\mathrm{W} \frac{\partial \mathrm{V}}{\partial \mathrm{Z}}\right]=-\frac{\partial \mathrm{P}}{\partial \mathrm{Y}}+\left[\frac{\partial^{2} \mathrm{~V}}{\partial \mathrm{X}^{2}}+\frac{\partial^{2} \mathrm{~V}}{\partial \mathrm{Y}^{2}}+\frac{\partial^{2} \mathrm{~V}}{\partial \mathrm{Z}^{2}}\right]$

$Z$ direction

$\operatorname{Re}\left[\mathrm{U} \frac{\partial \mathrm{W}}{\partial \mathrm{X}}+\mathrm{V} \frac{\partial \mathrm{W}}{\partial \mathrm{Y}}+\mathrm{W} \frac{\partial \mathrm{W}}{\partial \mathrm{Z}}\right]=-\frac{\partial \mathrm{P}}{\partial \mathrm{Z}}+\left[\frac{\partial^{2} \mathrm{~W}}{\partial \mathrm{X}^{2}}+\frac{\partial^{2} \mathrm{~W}}{\partial \mathrm{Y}^{2}}+\frac{\partial^{2} \mathrm{~W}}{\partial \mathrm{Z}^{2}}\right]$

Here $U, V, W$ are the velocities at $X, Y$ and $Z$ in non-dimensional form.

\subsection{Energy Formulation}

The energy equation for the fluid portion is as follows

$$
\operatorname{RePr}\left[\mathrm{U} \frac{\partial \theta}{\partial \mathrm{X}}+\mathrm{V} \frac{\partial \theta}{\partial \mathrm{Y}}+\mathrm{W} \frac{\partial \theta}{\partial \mathrm{Z}}\right]=\left[\frac{\partial^{2} \theta}{\partial \mathrm{X}^{2}}+\frac{\partial^{2} \theta}{\partial \mathrm{Y}^{2}}+\frac{\partial^{2} \theta}{\partial \mathrm{Z}^{2}}\right]
$$

The local Nusselt number is known as the ratio of the convective heat coefficient multiplied by the characteristic length over the water conductivity (i.e. $\frac{\mathrm{hD}}{\mathrm{k}}$ ) so based on the nondimensional adopted earlier it becomes the inverse of the temperature. Thus

$\mathrm{Nu}=\frac{1}{\theta}$ 
The performance evaluation criteria PEC is taken as the ratio between the average Nusselt number and the pressure drop to the power 0.3333 . Thus, the formulation for the PEC is as follows;

$$
\mathrm{PEC}=[\overline{\mathrm{Nu}}] /[\Delta \mathrm{P}]^{1 / 3}
$$

Here $\overline{\mathrm{Nu}}$ is the average Nusselt number for the current case under investigation. Similarly, the pressure drops $\Delta \mathrm{P}$ is for the pressure drop between the inflow located at the center of inlet mixing chamber and at the center of outlet mixing chamber.

\subsection{Boundary Conditions}

Figure 1 presents the boundary condition. The fluid enters with a temperature Tin which in non-dimensional form is $\theta$ set equal to 0 and an inlet velocity $u_{\text {in }}$ along the flow direction of 1 based on equation 1. Three different Reynolds numbers of 200, 400 and 600 have been investigated corresponding to an inlet flow rate of $7.56 \times 10^{-6} \mathrm{~m}^{3} / \mathrm{s}, 1.512 \times 10^{-5}$ $\mathrm{m}^{3} / \mathrm{s}$ and $2.268 \times 10^{-5} \mathrm{~m}^{3} / \mathrm{s}$ respectively. The flow rate is defined as the product of the inlet velocity multiplied by the cross-section area of the mixing chamber which is $0.0127 \mathrm{~m} \mathrm{x}$ $0.0375 \mathrm{~m}$. The system is heated from the bottom with a heat flux having a unit nondimensional value of 1 . At the outlet location of the flow a free boundary is applied. The entire external surfaces are assumed insulated to eliminate heat losses to the atmosphere.

\subsection{Mesh sensitivity analysis and convergence criteria}

The mesh sensitivity is examined in order to determine the optimal mesh required for the analysis. In Table 1, different mesh sizes were investigated following the terminology used by COMSOL software. The mesh levels that COMSOL supports and the elements numbers for each mesh level

Table 1 Mesh information for different level of meshing

Coarse

Normal

Fine
126890 domain elements, 17200 boundary elements, 1489 edge elements

232918 domain elements, 25988 boundary elements, 1859 edge elements

500224 domain elements, 43508 boundary elements, 2420 edge elements 


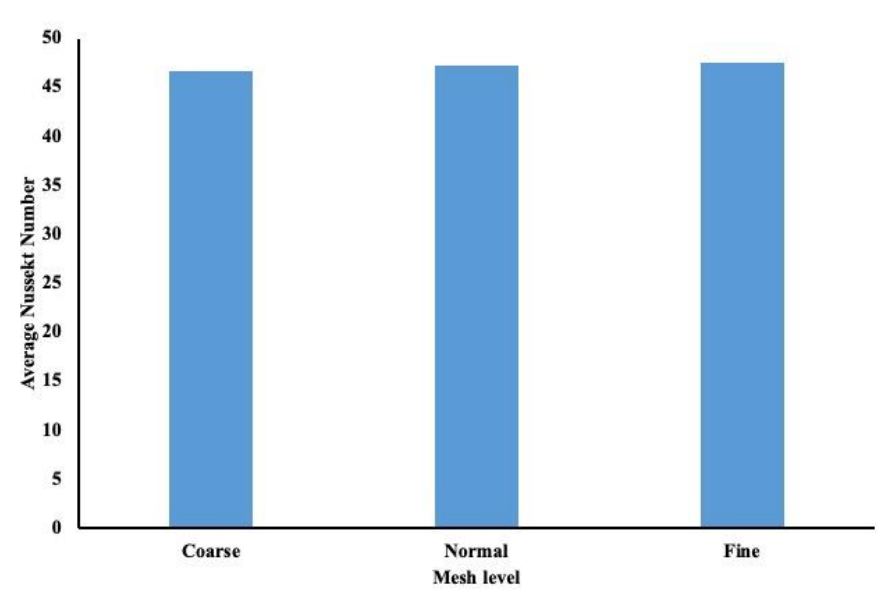

(a) Mesh sensitivity analysis

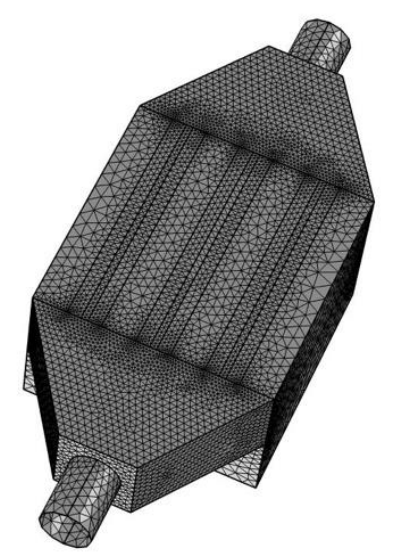

(b) Finite element model

Figure 3. Finite element analysis

are shown in Table 1. The average Nusselt number was evaluated at $1 \mathrm{~mm}$ below the interface in the Aluminum block and the results are represented in the Figure $3 a$. It is evident that a normal mesh level will be the suitable to be used. Figure $3 b$, presents the finite element mesh used in our simulation. Different approaches exist in COMSOL to tackle the convergence criteria. In this particular model the default solver used was the segregated method. Details about this approach could be found in any finite element's textbook. The convergence criterion is clearly explained in COMSOL manual. In a short summary the convergence criteria were set as follows: at every iteration, the average relative error of $U, V, W, P$ and $\theta$ were computed. These were obtained using the following relation:

$$
\mathrm{R}_{\mathrm{c}}=\frac{1}{\mathrm{n} \cdot \mathrm{m}} \sum_{\mathrm{i}=1}^{\mathrm{i}=\mathrm{m}} \sum_{\mathrm{j}=1}^{\mathrm{j}=\mathrm{n}}\left|\frac{\left(\mathrm{F}_{\mathrm{i}, \mathrm{j}}^{\mathrm{s}+1}-\mathrm{F}_{\mathrm{i}, \mathrm{j}}^{\mathrm{s}}\right)}{\mathrm{F}_{\mathrm{i}, \mathrm{j}}^{s+1}}\right|
$$

where F represents one of the unknowns, viz. $U, V, W, P$, or $\theta$, sis the iteration number, and $(i, j)$ represents the coordinates on the grid. Convergence is reached if $R_{c}$ for all the 
unknowns is below $1 \times 10^{-6}$ in two successive iterations. For further information on detailed solution method the reader is referred for COMSOL software manual [16].

\subsection{Comparison with experimental data}

Welsford et al [3] and Plant and Saghir [5,6] conducted experimental measurement of temperature in three channels configuration identical to the one shown in Figure 1. With respect to Welsford the channels were filled with metallic foam and the one conducted by Plant and Saghir were clear channels with no porous area. In both cases water was circulating at different flow rate and heating condition. Similar to our current cases, the temperatures were measured $1 \mathrm{~mm}$ below the interface as shown in Figure 1.

Figure 4 presents a comparison between the measured experimental data and the calculated numerical data. The three channels contained metallic foam insert having a permeability of 20 pores per square inches (20 PPI) which correspond to a permeability of $6.63304 \times 10^{-4} \mathrm{~m}^{2}$ and a porosity of 0.91 . The flow rate is set equal to 0.2 US Gallon Per Minute (USPM) corresponding to a flow rate of $1.26 \times 10^{-5} \mathrm{~m}^{3} / \mathrm{s}$. The Reynolds number in this particular comparison is $\mathrm{Re}=500$ and the Prandtl number Pr is equal to 6.8358 being water the used fluid. As shown in Figure $4 \mathrm{a}$ good agreement is achieved between the experimental and numerical data. As the flow circulate in the porous channel, the temperature increases accordingly. Figure $4 \mathrm{~b}$ shows the comparison for the Nusselt number between experimental and numerical. Some discrepancies are observed in the results near the entrance of the channel for which the flow may not be fully developed yet. However, a good agreement is observed as well. One may conclude from these results that the model is well calibrated to investigate the proposed current configuration. However, one may need to compare with clear channel case because the proposed model is a combination of porous strip and clear path.

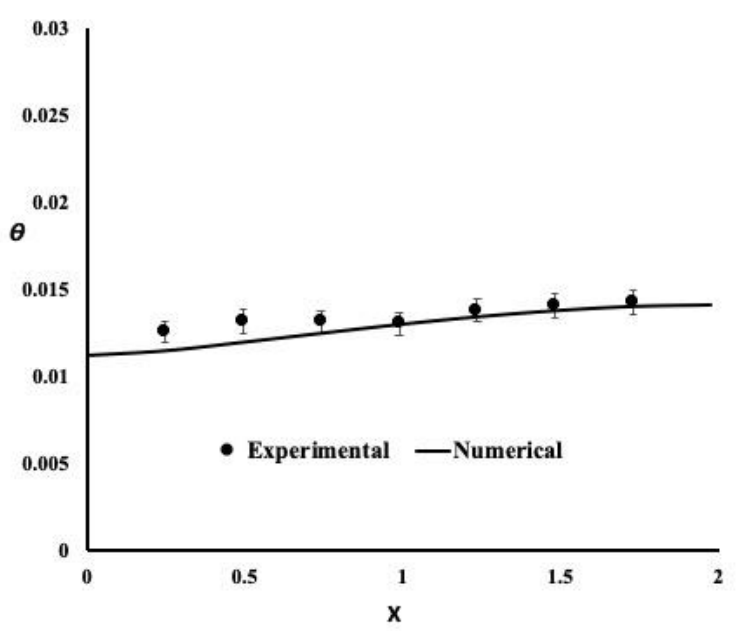

(a) Temperature distribution 


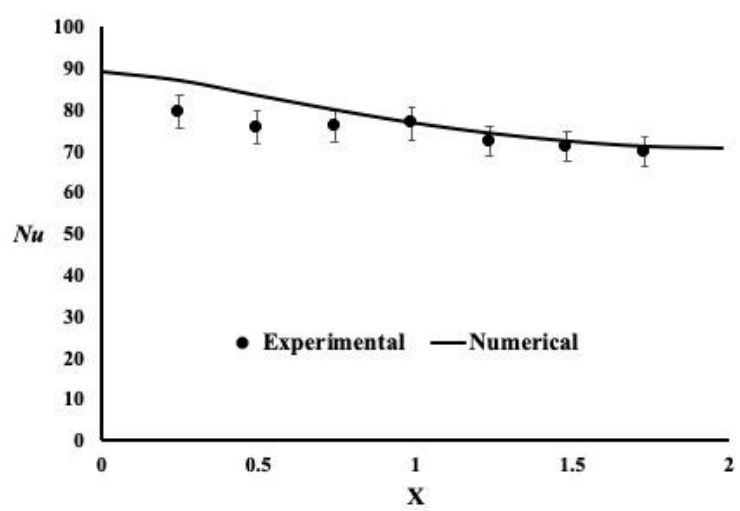

(b) Nusselt number variation

Figure 4. Comparison with experimental data for porous channels condition

Additional comparison has been conducted with similar case stated earlier but with free channels. Thus, the porous insert has been removed allowing the flow to move freely thus reducing the flow friction and the pressure drop. Plant and Saghir $[5,6]$ conducted an experiment studying heat enhancement in a free channel. Water is used as circulating fluid. The flow rate similar to the previous comparison was set at 0.2 US Gallon per Minutes for different heat fluxes applied at the bottom of the heated plate. Similar configuration setup as the current model is foreseen. The Reynolds number for this case is set at 500 . The inlet temperature was measured and found to be, depending on the heating condition, below 19 degrees Celsius.

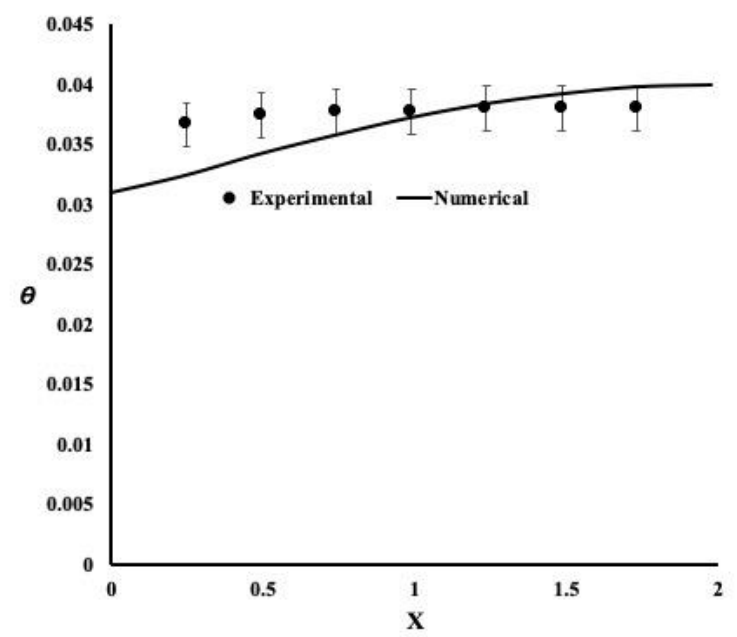

(a) Temperature distribution 


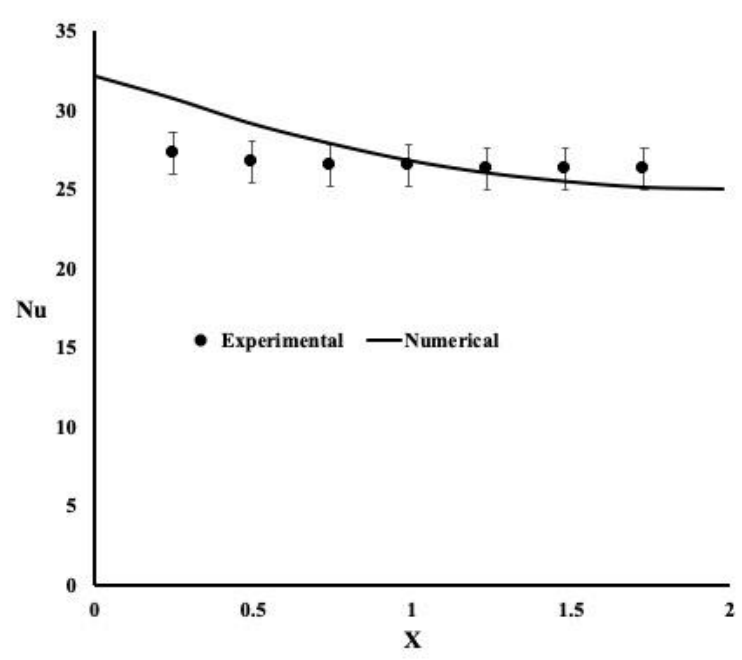

(b) Nusselt number variation

Figure 5. Comparison with experimental data for free channel case

Figure 5 displays the comparison between the two cases experimental and numerical. A good agreement is obtained between the two approaches. From Figure 4 and Figure 5 one may conclude that the numerical model is stable and accurate.

\subsection{Results and discussions}

In the previous section, we have demonstrated the accuracy of our numerical model in predicting precisely the temperature and the Nusselt number when compared to measured experimental data in our lab. The rational for choosing those two cases, is because in our study the insert is a combination of porous strip and free flow channel. Thus, the novelty and uniqueness of our current studied model. From previous cases we noticed that a porous medium can enhance the heat transfer at the expenses of large pressure drop. Whereas the opposite for free channel where heat enhancement is less than expected but with lower pressure drop. Hence, in the present study, attempt is made to study the optimum thickness of porous material for better heat enhancement and lower pressure drop.

Different cases have been investigated and the key parameter between each case is the so-called aspect ratio AR. This aspect ratio is the ratio of the thickness of the porous strip to the channel width. With constant channel width, the thickness of the porous strip varies from $0.000535 \mathrm{~m}$ to $0.002675 \mathrm{~m}$ which correspond to an aspect ratio varying between 0.1 to 0.5 respectively. When aspect ratio is set equal to 0.5 means that the entire channels are filled with porous foam. Contrary when the aspect ratio $A R$ is set equal to 0 means no porous strip in the channel which is identified as a free channel. 
Three different flow rates were investigated leading to a Reynolds number equal to 200, 400 and 600 respectively. For the porous strip, we have chosen a permeability of 20 pores per inches (i.e. 20PPI) which correspond to a permeability equal to $6.63304 \times 10^{-4} \mathrm{~m}^{2}$ and a porosity of 0.91 which are identical to our current setup and similar to our experimental comparison.

Figure 6 presents the temperature variation, calculated, $1 \mathrm{~mm}$ below the interface for different aspect ratio from 0.1 to 0.5 respectively.
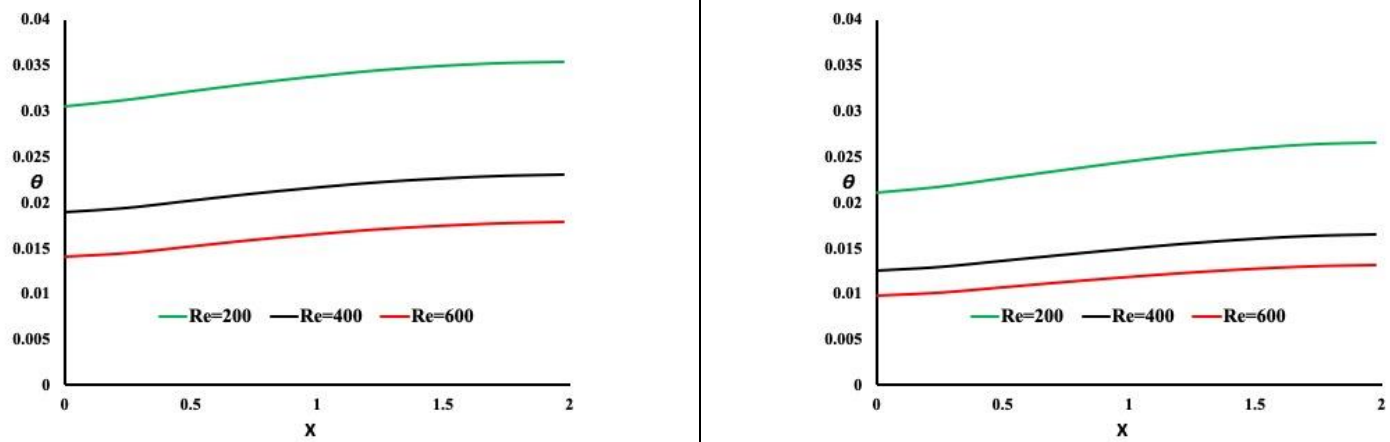

(a) $\mathrm{AR}=0.1$

(b) $A R=0.2$
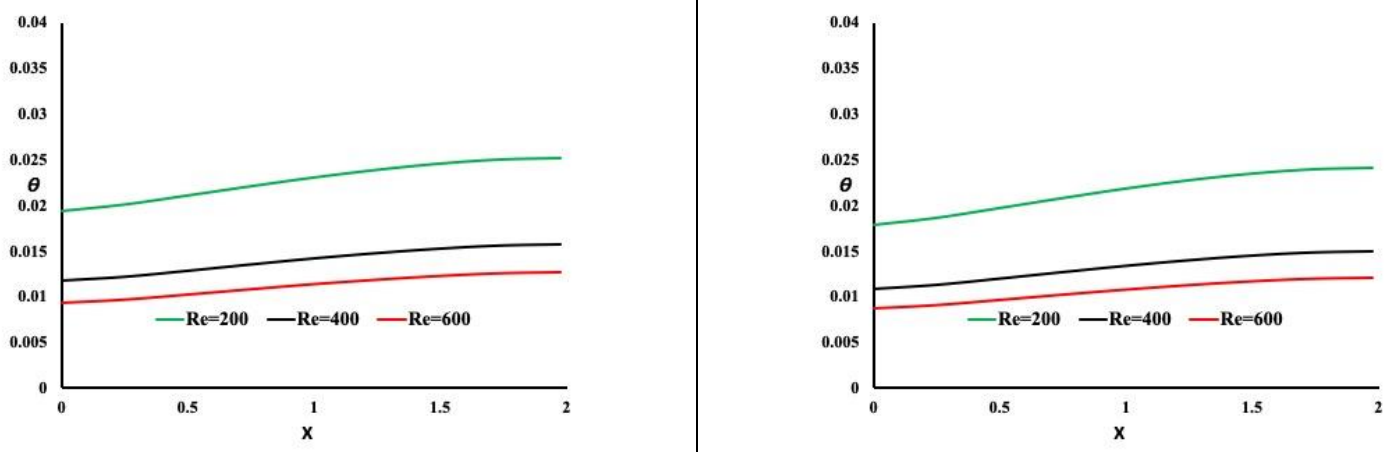

(c) $A R=0.3$

(d) $A R=0.4$

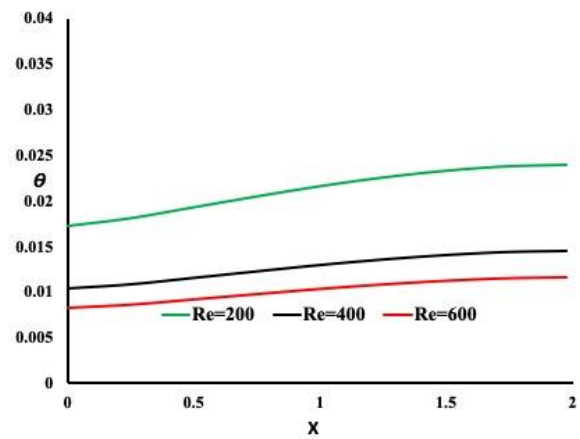

(e) $A R=0.5$ 
Figure 6 Temperature variation for different aspect ratio

It is clearly evident from the results in Figure 6 that as the aspect ratio increase, heat enhancement is noticeable. The temperature variation keeps dropping in magnitude as the porous material increase in the channels meaning the flow is absorbing more heat. Another obvious observation is that as the flow rate increase, the temperature drops as expected. The positive slope of the temperature is an indication that as the flow circulate in the channel heat extraction is less towards the end of the channel leading to a rise in temperature. This is due to the development of the boundary layer and thus forcing less heat to be extracted. Another way to observe this heat extraction phenomena is to display the Nusselt number variation along the flow path for different aspect ratio and flow rate as depicted in Figure 7.

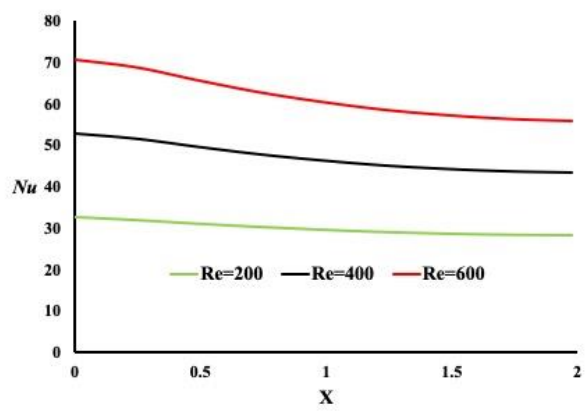

(a) $A R=0.1$

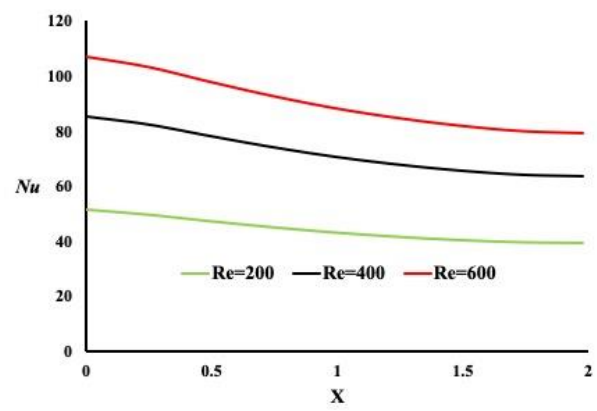

(c) $A R=0.3$

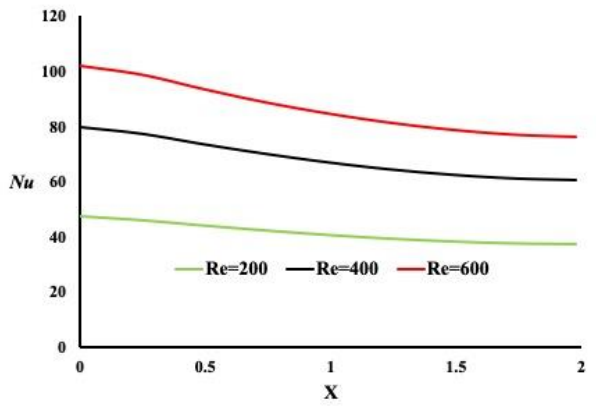

(b) $A R=0.2$

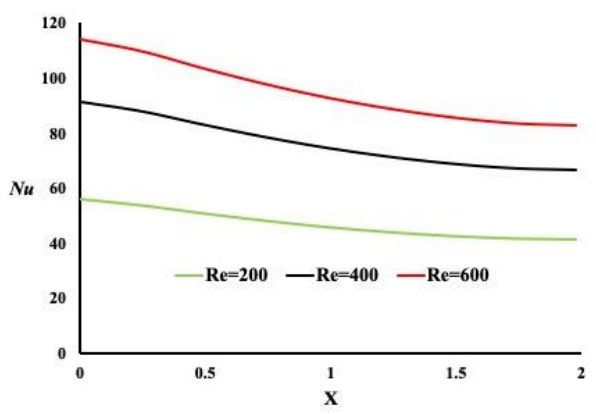

(d) $A R=0.4$ 


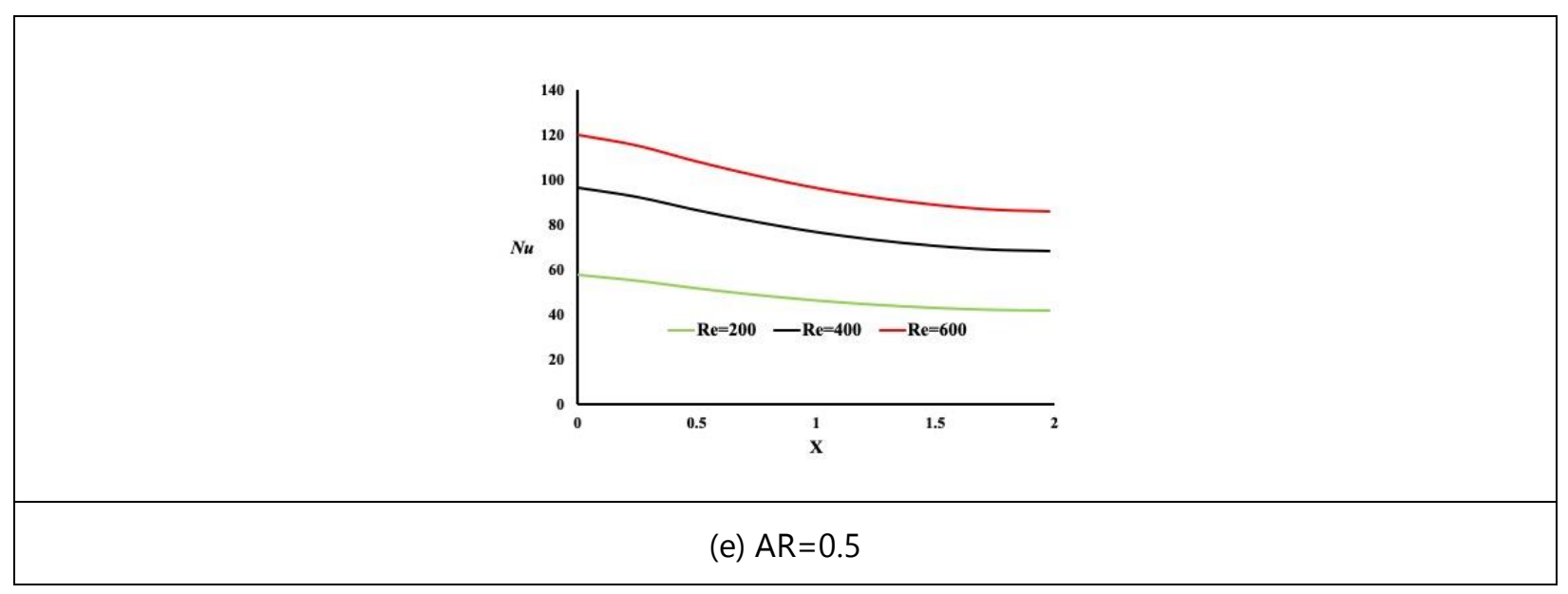

Figure 7 Nusselt number variation for different aspect ratio and flow rates

The variation trend of the Nusselt number regardless the cases studied is similar. It is the inverse of the non-dimensional temperature as indicated earlier. Also, it is evident that as the aspect ratio increase the Nusselt number increase as predicted. That mean heat enhancement is more noticeable in the presence of porous material. The volume of porous material in the channel is increasing leading to a better heat enhancement. The slope of the Nusselt number is a good indicator for the development of the thermal boundary layer at the entrance. As the flow progresses, the thickness of the boundary layer is increasing not allowing heat to dissipate into the flow. It is believed a combination of flow boundary layer and thermal boundary layer are implicated on heat dissipation to the fluid. Nevertheless, as Reynolds number increases the heat extraction increase accordingly.

Further investigation of the flow behaviour is needed in order to combine these two effects. Figure 8 presents the pressure drop variation between the flow inlet and the flow outlet for all cases from an aspect ratio of 0 to an aspect ratio of 0.5 . This pressure drop is a good indicator of the friction occurring during the flow in the channels. The position of the pressure drop is taken in the inlet mixing chamber and outlet mixing chamber thus taking into consideration the three channels effect. 


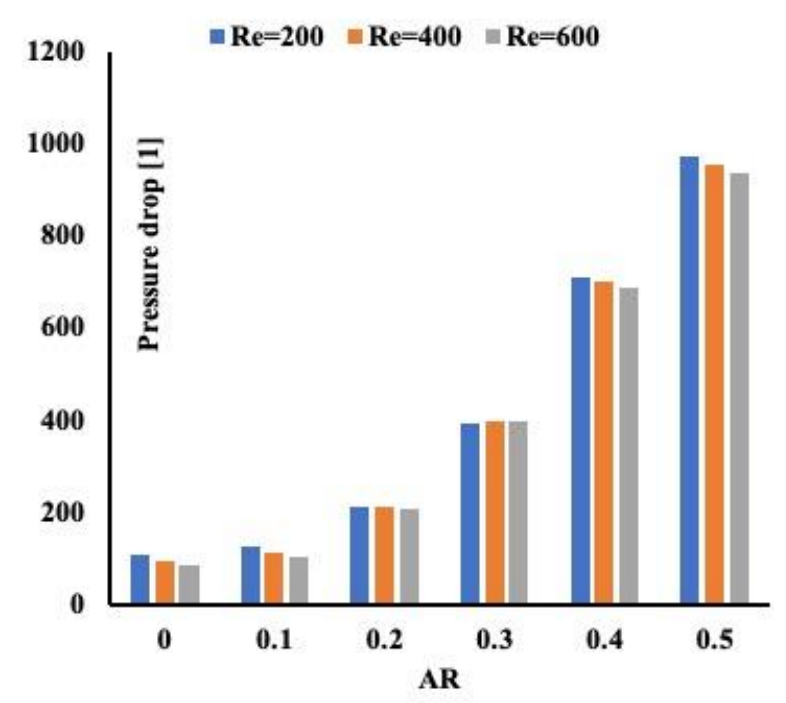

Figure 8 Pressure drop variation as function of Reynolds number and aspect ratio

The presence of porous strip regardless of its thickness (i.e. aspect ratio $A R$ ) generate a pressure drop larger than the case of the absence of porous strip (i.e. $A R=0$ ). Although it was noticed earlier that porous strip enhances the heat extraction but at the expenses of the pressure drop. However, as the flow rate represented by Reynolds number increase, a slight decrease in the pressure drop is observed as the aspect ratio increase. There must be an optimum aspect ratio or on other word porous strip thickness which can in one hand enhance the heat transfer and two lower the pressure drops. By combining the findings in Figure 7 and in Figure 8, Figure 9 presents the performance evaluation criteria defined in equation 8 for all cases.

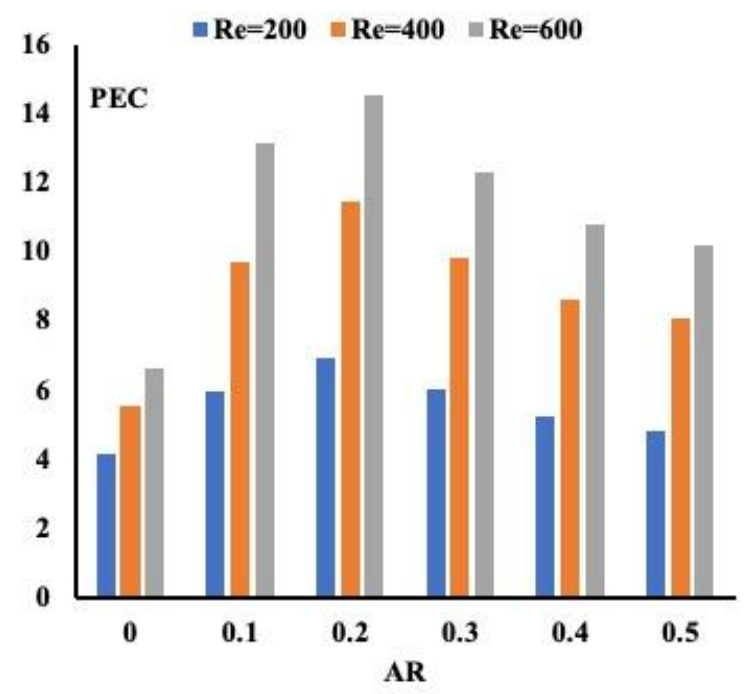

Figure 9 Performance evaluation criterion for all cases 
One may observe that by combining the heat enhancement and the flow behaviour we can achieve an optimum porous thickness which could be suitable for engineering application. As observed in Figure 9, with an increase in pressure drop due to the presence of porous strip, heat extraction is increasing as well. The performance evaluation criteria in Figure 9 shows that there is an optimum thickness to be considered. In our current case the optimum porous thickness corresponds to an aspect ratio of 0.2 . Before and after this aspect ratio, the performance of the channel decrease. This observation is valid for different Reynolds number as shown in Figure 9.

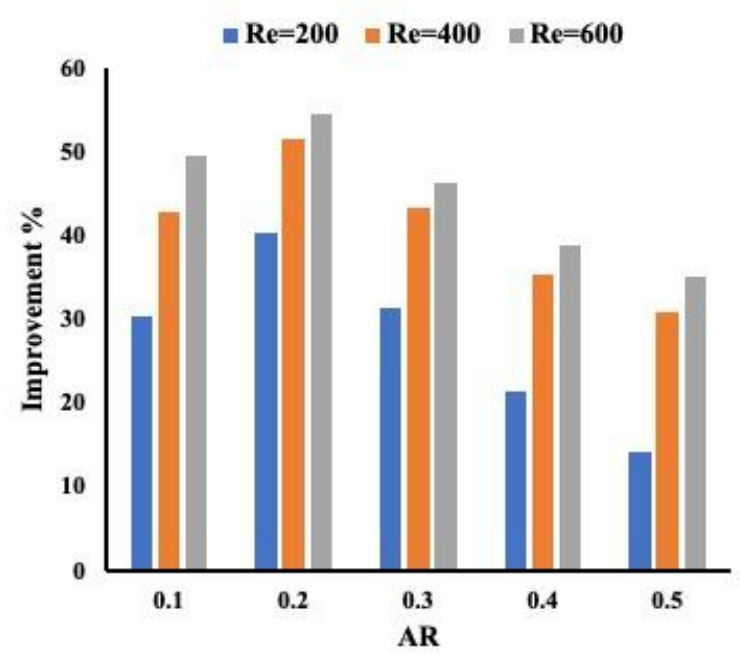

Figure 10 Performance evaluation criteria Improvement

Figure 10 presents the improvement percentage of the performance evaluation criteria when compared to the clear channels case. It is evident from Figure 10 that the best configuration is the one for an aspect ratio of 0.2. As the thickness of the porous layer increase a drop in the improvement is noticeable. This observation is applicable to all Reynolds number cases.

\subsection{Conclusion}

In our current study, we focussed our study on examining whether a porous strip in a channel can affect the heat extraction. Three channels of identical sizes were used and a mixing chamber at the inlet and at the outlet was modelled numerically using the finite element technique. By below heating condition, the goal consisted of determining whether an optimum porous strip thickness can be determined for heat enhancement. By comparing our numerical data with current experiment for the case of free channels and porous channel we demonstrated the accuracy of our model. In the 
present paper, the uniqueness of our study is the combination of porous material with free flow to determine the optimum porous thickness. Results revealed

1. The presence of porous material in a channel lead to a noticeable heat extraction at the expenses of pressure drop.

2. Comparison between experimental data and numerical results showed the robustness of our numerical code.

3. We have demonstrated that there is an optimum porous strip which can be used to extract the maximum heat for a minimum pressure drop. This optimum corresponds to an aspect ratio of 0.2 .

4. A maximum of $40 \%$ increase in performance evaluation criteria (PEC) is achieved for a Reynolds number equal to 200 and an aspect Ratio of 0.2 when compared to free channel. This improvement starts decreasing as the aspect ratio increase. The performance evaluation criteria increase as the Reynolds number increase to 400 and 600 which correspond to an increase of $51 \%$ and $54 \%$ respectively

Acknowledgement; This research was funded by [National Science and Engineering Research Council Canada, Faculty of Engineering and Architecture, Ryerson University] and [Qatar Foundation] grant number [NPRP12S-0123-190011]

\section{References}

1. Bayomy, A.M.; Electronic Cooling Using ERG Aluminum Foam Subjected to Steady/Pulsating Water $\mathrm{And}_{2} \mathrm{Al}_{2} \mathrm{O}_{3}-\mathrm{Water} \mathrm{Nanofluid}$ Flows: Experimental And Numerical Approach, PhD dissertation, Ryerson University

2. Bayomy, A.M; Saghir, M.Z. Thermal Performance of Finned Aluminum Heat Sink Filled with ERG Aluminum Foam: Experimental and Numerical Approach, International Journal of Energy Research, 2020, http://dx.doi.org/10.1002/er.5217.

3. Welsford, C. ; Thanapathy, P.; Bayomy, A.M.; Ren, M. Saghir, M.Z. Heat Enhancement using Aluminum Metal Foam: Experimental and Numerical Approach, Journal of porous media, 2020, Vol 23(3), pp 249-266,

4. Delisle, C. ;Welsford, C; Saghir, M.Z. Forced Convection Study with Micro-porous Channels and Nanofluid: Experimental and Numerical, J Therm Anal Calorim, 2019. https://doi.org/10.1007/s10973-019-09175-8

5. Plant, R.D; Hodgson, G.K.; Impellizzeri, S.; Saghir, M.Z. Experimental and Numerical Investigation of Heat Enhancement using a Hybrid Nanofluid of Copper Oxide/Alumina Nanoparticles in Water, J Therm Anal Calorim, 2020, Vo/ 141, pp 1951-1968

6. Plant, R.D; Saghir,M.Z, Numerical and Experimental Investigation of High Concentration Aqueous Alumina Nanofluids in a Two and Three Channel Heat Exchanger, International Journal of Thermofluids, 2021, Vol 9, 100055.

7. Alhajaj,Z; Bayomy, A.M; Saghir,M.Z; Rahman,M.M, Flow of Nanofluid and Hybrid Fluid in Porous Channels: Experimental and Numerical Approach, International Journal of Thermofluids, 2020 Vol 1-2, pp 1-14, 100016

8. Saghir, M.Z; Rahman,M.M, Forced Convection of $\mathrm{Al}_{2} \mathrm{O}_{3}-\mathrm{Cu}, \mathrm{TiO}_{2}-\mathrm{SiO}_{2}, \mathrm{FWCNT}^{-}-\mathrm{Fe}_{3} \mathrm{O}_{4}$ and $\mathrm{ND}-\mathrm{Fe}_{3} \mathrm{O}_{4} \mathrm{Hybrid}$ Nanofluid in Porous Media, Energies, 2020, Vol 13, 2902, doi:10.3390/en13112902,.

9. Saghir, M.Z; Welsford, $C$, Forced Convection in Porous Media Using $\mathrm{Al}_{2} \mathrm{O}_{3}$ and $\mathrm{TiO}_{2}$ Nanofluids in Differing Base Fluid: Experimental and Numerical approach, Energies, 2020, Vol 13, doi:10.3390/en131026.

10. Welsford, C; Delisle,C; Plant,R.D; Saghir, M.Z, Effects of Nanofluid Concentration and Channeling on the Thermal Effectiveness of Highly Porous Open-Cell Foam Metals: A Numerical and Experimental Study, J Therm Anal Calorim, 2019. https://doi.org/10.1007/s10973-019-09166-9 
11. Saghir, M.Z; Rahman, M.M. Brownian Motion and Thermophoretic Effects of Flow in Channels using Nanofluid: A Two-Phase Model, International Journal of Thermofluids, 2021, Vol 10,100085

12 Ghadikolaie,M.M; Saffar-Avval, M; Mansoori, Z; Alvandifar, N; Rahmati,N. Heat Transfer Investigation of a Tube Partially Wrapped by Metal Porous Layer as a Potential Novel Tube for Air Cooled Heat Exchangers, Journal of Heat Transfer,2019, Vol 141.

13. T'Joen, C.; De Jaeger, P.; Huisseune, H.; Van Herzeele, S.; Vorst, N.; De Paepe, M., Thermo-Hydraulic Study of a Single Row Heat Exchanger Consisting of Metal Foam Covered Round Tubes, Int. J. Heat Mass Transfer, 2010,53(15-16), pp. 3262-3274.

14. Al-Salem, K.; Oztop, H. F; Kiwan, S., Effects of Porosity and Thickness of Porous Sheets on Heat Transfer Enhancement in a Cross Flow Over Heated Cylinder, Int. Communications Heat Mass Transfer, 2011, 38(9), pp. 1279-1282.

15. Le Breton,P; Caltagirone, J.P; Arquis,E. Natural Convection in a Square Cavity with Thin Porous Layers on its Vertical Walls, Transaction of the ASME, 1991, Vol 113.

[16] COMSOL user manual, version 5.6, 2021, Newton, USA 